\title{
DESARROLLO DE DOS COLONIAS DE Bombus atratus (HYMENOPTERA: APIDAE) MANTENIDAS BAJO DOS MODOS DE ALIMENTACIÓN
}

\section{Comparative development of two Bombus atratus (Hymenoptera: Apidae) colonies with different feeding conditions}

Diego Riaño ${ }^{1,3} \bullet$ Manuel Veloza' • José Ricardo Cure $\bullet$ María Teresa Almanza ${ }^{2}$

\section{RESUMEN}

Bombus atratus es considerada una especie promisoria para ser implementada en programas de polinización de cultivos de tomate en Colombia. El método de cría utilizado para la producción de colonias era una adaptación del descrito para B. terrestris, Sin embargo, B. atratus difiere en el comportamiento de alimentación de las larvas (alimentación directa por bolsillo "pocket maker" vs. por regurgitación "pollen storer" en B.terrestris). Este trabajo describe de forma detallada el desarrollo de dos colonias criadas bajo dos condiciones de alimentación diferentes: a) cría cerrada, con ofrecimiento de néctar y polen por parte del asistente de laboratorio en la base de la caja de cría, semejante a la metodología descrita para B. terrestris (colonia cautiva - CC), b) cría cerrada hasta alcanzar diez obreras y con forrajeo externo a partir de ese momento (colonia forrajera - CF). La colonia CC produjo un total de 110 adultos (obreras+machos). El tiempo promedio de desarrollo para las obreras fue de $32,9 \pm 7.9$ y para los machos $35,7 \pm 9,1$ días. La mortalidad de larvas fue elevada (70\%). CF produjo 107 celdas de huevo de las que se generaron 344 obreras+machos. El tiempo promedio de desarrollo de las obreras fue $29,5 \pm 5.1$ y de los machos $30 \pm 6.8$ días. La mortalidad en el estado larval fue baja. En CF dos reinas silvestres tomaron el control de la colonia en momentos diferentes de su desarrollo. Los resultados del presente trabajo mostraron que la alimentación por regurgitación afecta la supervivencia de las larvas y por consiguiente el crecimiento y desarrollo de las colonias. Lo anterior permitió el desarrollo de técnicas combinadas de alimentación que permiten la cría de colonias de B. atratus en condiciones controladas.

Palabras clave: Abejorros, Cría de colonias, alimentación de colonias.

1 Grupo de Biodiversidad y Ecología de Abejas Silvestres, Facultad de Ciencias, Universidad Militar Nueva Granda. Km 2 vía Cajicá- Zipaquirá - Colombia.

2 Bayer CropScience, Product Development Manager.

3 Correo para correspondencia: diegorianojimenez@gmail.com; ecologiadeabejas@unimilitar.edu.co 
ABSTRACT

The native bumblebee Bombus atratus Franklin, 1913 has been found promissory to pollinate greenhouse tomato crops in Colombia. The initial rearing methodology for $B$. atratus was based on the methodology used in Europe for B. terrestris, nevertheless, B. atratus differ in larval feeding behavior (pocket maker vs. pollen storer in B.terrestris). This work describes detailed colony development for two colonies reared under two different feeding conditions: a) colony reared with provisioning of nectar and pollen on the floor of the rearing cage (Captive Colony-CC) (similar to the methodology used to rear B. terrestris), b) colony as in a) at the start of development but after reaching 10 adult workers with an open access to the field for foraging (Foraging Colony-CF). CC produced 183 egg cells that give rise to 110 adults (workers+males), the development time for workers was $32.8 \pm 7.9$

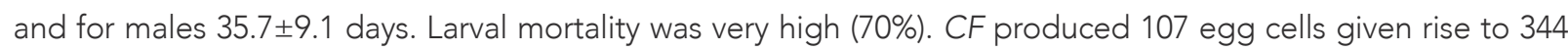
adults. Development time for workers was $29.5 \pm 5$ and for males $30 \pm 6.8$ days. Mortality was low in the larval stage. Two wild queens took control of CF colony at different times of colony development. Implications of the two feeding ad rearing methods for successfully $B$. atratus colony development are discussed.

Keywords: bumblebee, colony rearing, colony feeding.

\section{INTRODUCCIÓN}

Con el propósito de mitigar el impacto de la melitofauna foránea utilizada para polinizar cultivos de importancia económica en las poblaciones de abejas y ecosistemas nativos, muchos países iniciaron la búsqueda de abejas nativas con potencial para ser incluidas en programas de polinización de cultivos (Asada \& Onno 1996, Asada 1997, Estay et al. 2001, Whittington \& Winston 2004, Palma et al. 2008). La facilidad de cría, la capacidad de adaptación a los cultivos y un efecto significativo en la producción son algunos de los aspectos que deben ser considerados para que una especie tenga potencial como agente polinizador de cultivos comerciales. Bombus atratus Friese 1913 es una especie con una amplia distribución en Suramérica desde Colombia hasta Argentina, presenta una alta adaptabilidad a ambientes perturbados, tiene un amplio rango de distribución (desde 150 a 3200 msnm) (González et al. 2004), presenta actividad constante durante todo el año y tiene varios ciclos de producción de sexuados (reinas y machos) (Silva-Matos \& Garofalo 1995). Estas características han despertado gran interés en la cría de colonias de B. atratus para implementarlas como estrategia de polinización de cultivos para Suramérica (Salvarrey et al. 2013). En Colombia, el grupo de Biodiversidad y Ecología de Abejas Silvestres de la Universidad Militar Nueva GranadaBogotá ha desarrollado una metodología de cría de colonias de $B$. atratus y ha adelantado estudios en polinización dirigida de cultivos con colonias de $B$. atratus los cuales han demostrado la eficiencia de ésta especie como polinizador (Aldana et al. 2007; Almanza 2007). El desarrollo del sistema de cría ha tenido que superar algunas limitaciones (Almanza 2007), teniendo la alimentación de las colonias como uno de los factor más limitantes. 
Los ensayos iniciales de cría se realizaron con la metodología desarrollada para Bombus terrestris (L.) en Europa (van den Eijnde et al.1991), en donde las colonias se alimentan con una mezcla de polen y solución azucarada dispuesta libremente cerca de la colonia. Posteriormente el polen es recogido por las obreras y almacenado en ollas de cera para su posterior uso. Sin embargo las colonias de B. atratus criadas bajo esta metodología no mostraban un crecimiento y desarrollo normal comparadas con lo observado en colonias silvestres (obreras de tamaño pequeño, baja producción de obreras y de sexuados). Según Ptacek \& Drobna (2006) uno de los factores que se debe tener en cuenta para la cría de especies del género Bombus es la forma en que las obreras alimentan a las larvas. Según el tipo de alimentación, las especies del género Bombus pueden ser diferenciadas en almacenadoras de polen "pollen storers" o constructoras de bolsillos "pocket makers". B. terrestris es una especie que almacena el polen en pequeñas vasijas de cera (Velthius \& van Doorn 2006). Cuando las larvas necesitan ser alimentadas, las obreras toman el polen almacenado y lo regurgitan sobre las larvas (Katayama 1973, Hannan et al. 1998). En contraste B. atratus es una especie constructora de bolsillo, estructura por la cual las obreras alimentan las larvas introduciendo el polen en los bolsillos y poniéndolo a disposición de las larvas inmediatamente después de ser recolectado (Sakagami et al. 1967).

Teniendo en cuenta estas diferencias y con el propósito de establecer el efecto del modo de alimentación en el desarrollo de las colonias (tiempo de desarrollo de los individuos, número de individuos producidos y tasa de crecimiento de las coIonias) se realizaron observaciones diarias por un periodo de 160 días a dos colonias de B. atratus, una mantenida bajo el esquema de alimentación adaptado de la metodología de cría de B. terrestris (Colonia Cautiva CC) y la otra bajo condiciones de semicautiverio, y en la cual las obreras podían forrajear y alimentar sus larvas de forma natural (Colonia Forrajera CF).

\section{MATERIALES Y MÉTODOS}

\section{Origen y manejo de las colonias}

Se colectaron 12 reinas de B. atratus en el Campus de la Universidad Militar Nueva Granada (Cajicá - Colombia). Las reinas fueron capturadas con redes entomológicas y puestas individualmente en recipientes de vidrio con tapas que permitían la entrada de aire. Inmediatamente después se llevaron a una cámara de cría climatizada ubicada en el mismo Campus $\left(26-28^{\circ} \mathrm{C}\right.$ y H.R. 35 y $\left.40 \%\right)$. Una vez en la cámara de cría, cada reina se ubicó en una caja de dimensiones $15 \times 10 \times 10 \mathrm{~cm}$ con una cara frontal de vidrio y estructura de madera de acuerdo al protocolo de iniciación de reinas implementado en nuestro laboratorio durante cerca de ocho años.

De las 12 reinas colectadas tan solo dos produjeron colonias, las diez reinas restantes no pusieron huevos o murieron prematuramente y por esa razón no fue posible incluirlas en las observaciones. Al emerger las primeras obreras, las dos colonias originadas de esas reinas se trasladaron a cajas de mayor tamaño $(30 \times 20 \times 20 \mathrm{~cm})$, con dos caras laterales de vidrio que facilitaron las observaciones.

\section{Variables evaluadas}

Cada una de las dos colonias estudiadas se rotuló de acuerdo al proceso de cría a que fue sometida: Colonia Cautiva (CC) y Colonia Forrajera (CF).

CC se mantuvo confinada en la cámara de cría desde su iniciación hasta el final de su ciclo, poniendo a disposición de la colonia solución azucarada (1:1 v/p agua y sacarosa) y tiras de polen fresco (30 g.) proveniente de colonias de Apis mellifera humedecido con la solución azucarada cada tercer día. Esta metodología obligaba a que las obreras recogieran 
hasta el final de las observaciones. Adicionalmente

y regurgitaran el polen para alimentar las larvas. Este procedimiento se utilizó a lo largo de todo el proceso de desarrollo de la colonia.

CF fue mantenida en la cámara de cría y alimentada de la misma manera que CC hasta que alcanzó 10 obreras (día 31 después de que la reina inició la oviposición). Posteriormente se le permitió forrajear mediante un tubo de PVC de 0,5" incrustado en las paredes de la cámara de cría que conectaba el interior de la colonia con el exterior para que las obreras pudieran salir y colectar polen en los jardines del Campus. A esta colonia se le siguió administrando solución azucarada diariamente durante el resto de las observaciones, pero no se le ofreció polen.

\section{Registro de datos}

Durante 160 días se realizó el conteo diario de las celdas en los diferentes estadios de desarrollo y los adultos encontrados en las dos colonias. Cada celda corresponde a un grupo de huevos puestos al mismo tiempo en una estructura de cera que crece a medida que se desarrollan los individuos. Al final del estadio larval y en el estadio de pupa, cada individuo de la celda se encuentra de forma individual. Para discriminar el estadio en el que se encontraba cada una de las celdas se tuvo en cuenta su apariencia de la siguiente forma: celdas esféricas de cera de color marrón oscuro y diámetro no mayor a $5 \mathrm{~mm}$ corresponde al estadio de huevos; celdas con una estructura de cera en forma de bolsillo aparece cuando se inicia el estado larval (Fervidobombus); celdas en que se distinguen claramente los individuos de la celda, se pierde el bolsillo de cera y tienen una coloración más clara que las anteriores corresponde al estadio de pupa. Los adultos encontrados también se contaban y se registraban clasificándose como obreras, nuevas reinas o machos.

La dinámica de crecimiento de las colonias se determinó a partir de la acumulación de las celdas en el estadio de huevo, larva y pupa, y al número de adultos producidos (obreras, machos o nuevas reinas) se diferenciaron las fases descritas para esta especie.

Se calculó y comparó el tiempo de desarrollo de los individuos producidos en CC y CF con la prueba estadística F y T (programa estadístico R, versión Macintosh 2.9.1).

El porcentaje de mortalidad $\left(M_{j}\right)$ de las celdas en cada estadio de desarrollo ( $i=1$, huevos, $i=2$, larvas, $i=3$, pupas) se calculó a partir de la siguiente fórmula:

\section{$\mathbf{y}=\mathbf{1} \mu+\mathbf{z}_{1} \mathbf{f}+\mathbf{z}_{2} \mathbf{h}+\mathbf{z}_{\mathbf{3}} \mathbf{b}$}

Donde $N_{i}$ corresponde al número acumulado de celdas en el estadio inicial y $N_{i+1}$ corresponde al número total de celdas que pasaron al siguiente estadio.

\section{RESULTADOS}

\section{Crecimiento de las colonias}

En las dos colonias se consideran separadamente sus fases monogínica (una sola reina) (Fig. 1a) y de competencia (cuando las obreras inician oviposición) (Fig. 1b), con la aclaración de que la colonia CF presentó un estado intermedio en donde la reina original fue substituida por una reina invasora y ésta a su vez fue substituida por una tercera reina, habiéndose presentado cortos períodos de convivencia de dos reinas durante este período (período de invasión) (Fig. 1c). Las curvas de crecimiento para CC y CF fueron muy diferentes.

CC: Durante la fase monogínica se presentó un rápido crecimiento en el número de celdas construidas por la reina, alcanzando 183 celdas, sin embargo, el número de adultos de allí resultantes (obreras+machos+nuevas reinas) fue muy bajo, emergieron apenas $98+5+1$ indivíduos (0,57 adultos por celda). Durante la fase de competencia, en ausencia de la reina, las obreras construyeron 48 celdas que resultaron en apenas seis machos adultos ( 0.13 adultos por celda) (Tabla 1$)$. En las dos fases descritas en CC, las celdas en estadio larval fueron las que presentaron la mortalidad más alta (Tabla 1). 

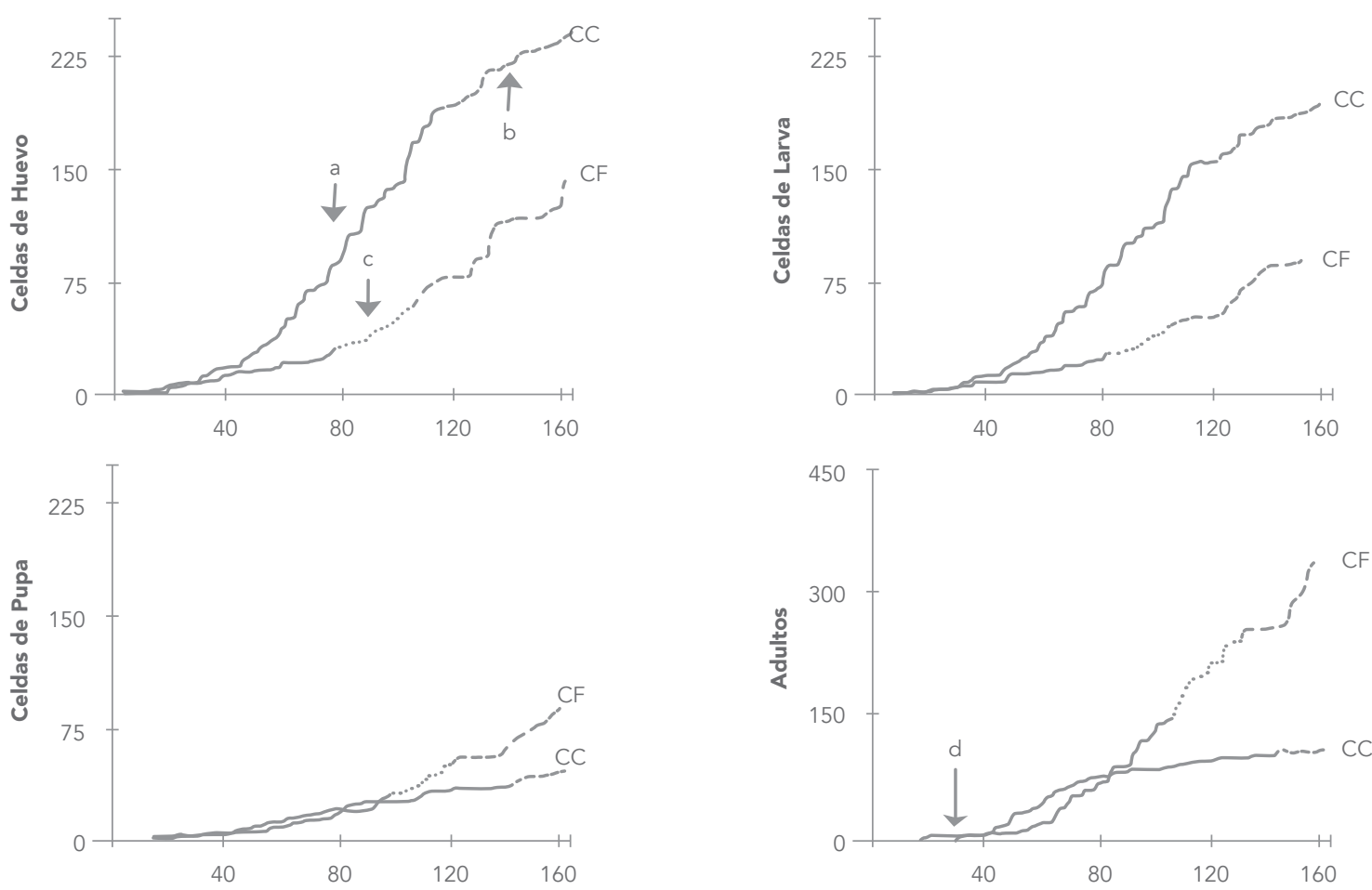

Días despues de la producción de la primera celda de huevo

Figura 1. Acumulación de celdas durante las fases monogínica (a) y de competencia (b) en la colonia cautiva (CC) y en la colonia forrajera (CF). Las diferentes puntuaciones de las curvas indican el número de celdas o el número de adultos generados a partir de posturas realizadas durante cada una de las fases y el periodo de invasión (c); por esta razón se ve un desplazamiento entre las diferentes texturas de las curvas según su estado de desarrollo. (d) indica el día en el que se le permitió forrajear a CF.

CF: Durante la fase monogínica la construcción de celdas estuvo por debajo de la observada en CC, alcanzado 29 celdas de huevo en 76 días, sin embargo de estas se originaron 149 obreras (5,14 adultos por celda), antes del período de invasión, momento en que la reina original fue eliminada por la primera reina invasora (día 79). Esta segunda reina a su vez fue eliminada por una tercera reina invasora, a los 86 días. Este período de invasión tuvo una duración de 21 días durante los cuales se originaron 25 celdas de las que emergieron 96 obreras adicionales (5,96 adultos por celda), antes de entrar a la fase de competencia. Esto indica que durante el período de invasión la colonia continuó su desarrollo casi sin haber sido afectada en su dinámica. La fase de competencia inició en el día 101, con la muerte de la segunda reina invasora. En esta fase fueron construidas 98 nuevas celdas por las obreras en 56 días. De las celdas producidas hasta el último día de observaciones, 19 habían finalizado su desarrollo y originaron 98 machos (5,15 machos por celda). Durante esta fase se presentó un incremento de la mortalidad en los estadios de huevo y larva en comparación con las otras dos fases, sin embargo nunca excedió lo observado en la colonia CC (Tabla 1). Es importante anotar que después de finalizadas las observaciones detalladas, este nido produjo varias nuevas reinas y machos, por lo que puede afirmarse que fue un nido completamente exitoso a pesar de la secuencia de invasiones y substitución de reinas por las que pasó. 
Tabla 1. Número de celdas en cada estadio de producidas en CC y CF

\begin{tabular}{cccccccc}
\hline Colonia & Fase & Huevo $^{++}$ & Larva $^{++}$ & Pupa $^{++}$ & Obreras & Machos & Reinas \\
\hline CC & Monogínica & $183(21.3)$ & $144(70.8)$ & $42(2.4)$ & 98 & 5 & 1 \\
\hline CC & Competencia $^{+}$ & $48(56.3)$ & $21(66.6)$ & $7(28.6)$ & 0 & 6 & 0 \\
\hline CF & Monogínica & $29(0)$ & $24(17.2)$ & $24(0)$ & 149 & 0 & 0 \\
\hline CF & período de invasión $^{\star}$ & $25(24)$ & $19(10.5)$ & $17(0)$ & 96 & 0 & 0 \\
\hline CF & Competencias $^{5}$ & $53(45.3)$ & $29(34.5)$ & $19(0)$ & 0 & 98 & 0 \\
\hline
\end{tabular}

*Ingreso de reinas foráneas durante la fase monogínica

+ Para la fase de competencia solo se tuvieron en cuenta las celdas que habían culminado su desarrollo o habían muerto al final de las observaciones (53 celdas).

++ Número de celdas y (\% de mortalidad)

Tiempo de desarrollo de los adultos de B. atratus.

El tiempo total de desarrollo de las obreras de $B$. atratus difirió entre las colonias CC y CF (Prueba T, $p<0.001)$. En la colonia $C C$ fue de 32.8 días $(D E=7.9$ días) y para CF fue de 29.5 días ( $D E=5$ días). En el caso de los machos, el tiempo de desarrollo también se diferenció entre las dos colonias (Prueba T, $\mathrm{P}<0.05$ ). En la colonia CC el tiempo de desarrollo promedio fue de 35.7 días ( $D E=9.1$ días) y en la colonia CF fue de 30 días ( $\mathrm{DE}=6.8$ días) (Tabla 2).

Tabla 2. Tiempo de desarrollo de cada estadio de las obreras y machos de B. atratus.

\begin{tabular}{ccccccccc}
\hline & \multicolumn{4}{c}{ Obreras } & \multicolumn{4}{c}{ Machos } \\
\hline & Huevo & Larva & Pupa & $\mathbf{n}$ & Huevo & Larva & Pupa & $\mathbf{n}$ \\
\hline CC (DE) & $7.8(1.7)$ & $14.5(5.0)$ & $10.8(6.1)$ & 98 & $8.2(2.4)$ & $19.0(6.7)$ & $8.6(3.9)$ & 11 \\
\hline$C F(D E)$ & $6(2.6)$ & $13.9(4.4)$ & $8.7(3.6)$ & 246 & $6.1(2.0)$ & $14.3(5.2)$ & $9.5(3.1)$ & 98 \\
\hline$P$ & 0.0021 & 0.13 & 0.00007 & & 0.01 & 0.02 & 0.2 & \\
\hline
\end{tabular}

n corresponde al número de individuos tenidos en cuenta para el cálculo del tiempo de desarrollo. (DE)

\section{DISCUSIÓN}

El crecimiento de las colonias de Bombus depende de la disponibilidad de recursos en el ambiente (polen y néctar) (Pelletier \& McNeil, 2003). A partir de la construcción de la primera celda las dos colonias iniciaron su crecimiento con el mismo vigor por lo menos hasta el día 31, cuando CF tuvo acceso al forrajeo externo y dejó de suministrársele polen artificialmente. La alta producción de celdas observada en CC pudo estar relacionada con la provisión constante de recursos (solución azucarada y polen) en la cámara de cría. En contraste, el crecimiento pausado observado en CF estuvo determinado por la disponibilidad de recursos florales en el ambiente y el gasto energético debido a su adquisición, variables que no existían en CC. 
Bajo condiciones naturales, las obreras de $B$. atratus alimentan las larvas ubicando directamente en los bolsillos de alimentación el polen recolectado de las flores, tal como se observó en la colonia CF. En el caso de la colonia CC, a la que se administró el polen cada tercer día, las obreras de la colonia alimentaron a las larvas regurgitando el polen suministrado. El comportamiento de alimentación por regurgitación aunque no es normal en $B$. atratus, permite el desarrollo de las colonias a pesar de que no muestren un crecimiento optimo en términos del tamaño de la colonia, producción de sexuados y tamaño de los individuos (Cruz et al. 2008, Almanza incluyendo B. atratus y está determinado tanto por el aumento de los requerimientos proteicos de las obreras necesarios para la maduración de los ovarios como por la competencia reproductiva entre ellas (Silva-Matos \& Garòfalo 1995, Cameron \& Jost 1998, Bourke \& Ratnieks 2000, Foster et al. 2004).

El estrés por el confinamiento en CC, debido al incremento del número de individuos en las cajas de cría, es un factor adicional que debe tener un efecto sobre el cuidado y la supervivencia de los estados inmaduros en desarrollo (Cnaani et al. 2002, Smeets \& Duchateau 2003, Foster et al. 2004). Este aspecto puede explicar el tiempo de desarrollo de las obre-

\section{A partir de los resultados obtenidos se pudo evidenciar que las colonias mantenidas bajo un esquema de cría en cautiverio y alimentada bajo el esquema de B. terrestris (CC) presenta una producción de huevos mucho mayor y más rápido que las colonias mantenidas en condiciones semi-naturales (CF) durante la fase monogínica.}

2007). En este sentido, el estadio larval en CC mostró el mayor porcentaje de mortalidad. El modo de alimentación por regurgitación observado pudo afectar la acumulación del polen dentro de las celdas larvales afectando la supervivencia de las mismas y en consecuencia el crecimiento de esta colonia.

En las dos colonias, después de la muerte de la reina fundadora aumentó el descarte y consumo de los huevos (oofagia), la eyección de larvas y el descuido de las celdas (incubación y alimentación) por parte de las obreras. Esto ha sido reportado anteriormente en diferentes especies del género Bombus, ras y machos de CC, similar a lo obtenido anteriormente bajo las mismas condiciones de cría (32.7 días) (Almanza 2007). En CF, el tiempo de desarrollo de los adultos fue similar al reportado en condiciones silvestres (obreras 29.6 días y machos 30 días) (Zucchi 1973; González et al. 2004).

Aunque el efecto del comportamiento de invasión de las reinas ("drifting") foraneas no era parte de los objetivos del estudio, se incluyeron debido a que es el primer registro de éste comportamiento, diferente al reportado por Cameron y Jost (1998) quienes describieron que reinas hijas de la reina 
fundadora podían tomar un territorio dentro de la colonia luego de que se apareaban (fase poligínica). El comportamiento de invasión ha sido reportado anteriormente en obreras de A. mellifera, B. occidentalis y B. impatiens (Birmingham et al. 2004). Según Birmingham et al. (2004), el comportamiento de invasión puede aumentar la capacidad reproductiva ("fitness") de los individuos invasores (parasitismo social). Lo anterior puede ser corroborado debido a que las dos reinas invasoras que se establecieron en la colonia CF y que lograron poner huevos, se favorecieron de las obreras presentes en la colonia (incubación, termorregulación, forrajeo). Esto permitió una alta tasa de oviposición de las reinas invasoras (25 celdas), que produjeron 96 nuevas obreras, en un periodo de tiempo menor al que tuvo la reina fundadora (Reina Fundadora 76 días, 29 celdas, 149 obreras, 5,14 obreras/celda; Reinas Invasoras 21 días, 5,96 obreras/celda). Éste comportamiento debe ser explorado con mayor detalle.

A partir de los resultados obtenidos se pudo evidenciar que las colonias mantenidas bajo un esquema de cría en cautiverio y alimentada bajo el esquema de B. terrestris (CC) presenta una producción de huevos mucho mayor y más rápido que las colonias mantenidas en condiciones semi-naturales (CF) durante la fase monogínica, aspecto que se ha podido evidenciar en otros estudios del laboratorio (datos sin publicar). Sin embargo el modo de alimentación por regurgitación de las larvas disminuye considerablemente la viabilidad de las larvas, afectando por consiguiente el crecimiento de la colonia. Por otra parte la colonia CF tuvo una baja producción de huevos durante su fase monogínica, sin embargo el número de individuos por celda fue mucho mayor que el observado en CC, debido probablemente a que el comportamiento de alimentación vía bolsillo permite que cada larva tenga acceso a una mayor cantidad de polen, aspecto que se reflejó en la baja mortalidad observada en este estadio. Las observaciones realizadas fueron la base para el desarrollo de una metodología combinada de alimentación que disminuye el tiempo de alimentación y permite el desarrollo de colonias de tamaño medio en un periodo de tiempo corto.

\section{AGRADECIMIENTOS}

Al Fondo de Investigaciones de la Universidad Militar Nueva Granada por la financiación del proyecto, a Alexander Escobar, Paola Cruz y Carlos Alberto Garófalo por sus importantes aportes y comentarios. Al personal de campo de la Facultad de Ciencias Básicas y Aplicadas por su valiosa colaboración. 


\section{REFERENCIAS}

1. Aldana J., J.R Cure, M.T. Almanza, D. Vecil \& D. Rodríguez. 2007. Efecto de Bombus atratus (HYMENOPTERA: APIDAE) sobre la productividad de tomate (Lycopersicum esculentum Mill) bajo invernadero en la Sabana de Bogotá, Colombia. Agronomía Colombiana. 25: 62-72.

2. Almanza M. 2007. Management of Bombus atratus bumblebees to pollinate Lulo (Solanum quitoense L.), a native fruit from the Andes of Colombia. Ecology and Development series. 50: 1-112.

3. Asada S. \& M. Ono. 1996. Crop pollination by japanese bumblebees Bombus spp. (Hymenoptera: Apidae): Tomato foraging behavior and pollination efficinecy. Appl. Entomol. Zool. 31: 581-586.

4. Asada S. \& M. Ono. 1997. Tomato pollination with japanese native bumblebees (Bombus spp.). Acta Hort. 437: 289-292.

5. Birmingham A. \& M. Winston. 2004. Orientation and drifting behaviour of bumblebees (Hymenoptera:Apidae) in comercial tomato greenhouses. Can. J. Zool. 82: 52-59.

6. Bourke A. F. G. \& F.L. Ratnieks. 2000. Kin-selected conflict in Bombus terrestris (Hyme noptera: Apidae). Proc. R. Soc. Lond. 26: 347-355.

7. Cameron S. \& M. Jost. 1998. Mediators of dominance and reproductive success among queens in the cyclically polygynous Neotropical bumble bee Bombus atratus Franklin Insectes soc. 45: 135-149.

8. Cnaani J., R. Schmid-Hempel, \& J. SchmidHempel. 2002. Colony development, larval development and worker reproduction in Bombus impatiens Cresson. Insectes soc. 49: 164-170.
9. Cruz P., A. Escobar, M.T. Almanza \& J.R. Cure. 2008. Mejoras para la cría en cautiverio de colonias del abejorro nativo Bombus atratus ( $=B$. pauloensis) (Hymenoptera: Apoidea). Revista Facultad de Ciencias, Universidad Militar Nueva Granada. 4: 70-83.

10. De Ruijter, A. 1997. Commercial bumblebee rearing and its implications. Acta Hortic. (ISHS). 437: 261-270.

11. Estay P., A. Wagner \& M. Escaff. 2001. Evaluation of Bombus dahlbomii (Guér.) as a pollination agent for tomato (Lycopersicon esculentum (Mill.)) flowers under greenhouse conditions. Agric. Tec. 61: 113-119.

12. Foster R., A. Brunskill, D. Verdirame \& S. O 'donnell. 2004. Reproductive physiology, dominance interactions, and division of labour among bumble bee workers. Physiological Entomology. 29: 327-334.

13. Gonzalez V., A. Mejia \& C. Rasmussen. 2004. Ecology and Nesting Behavior of Bombus atratus Franklin in Andean Highlands (Hymenoptera: Apidae). J. Hym. Res. 13: 234-242.

14. Hannan A., Y. Maeta \& K. Hoshikawa. 1998. Feeding behavior and food consuption in Bombus (Bombus) ignitus ander artificial condition (Hymenoptera: Apidae). Ento. Scien. 1: 27-32.

15. Kaftanoglu O. 2000. The Diversity and Faunistics of Bumble Bee for Pollination in Greenhouses. Insect Pollination in Greenhouses. Netherlands, 30 september to 2 october. 73-83.

16. Katayama E. 1973. Observations on the brood development in Bombus ignitus (Hymenoptera: Apidae) II: Brood development and feeding habits. Kontyú. 41: 203-216. 
colonies of Bombus atratus (Hymenopters: Apidae). Journal of apicultural research 34: 177-185.

26. Smeets P. \& J. M. Duchateau. 2003. Longevity of Bombus terrestris workers (Hymenoptera: Apidae) in relation to pollen availability, in the absence of foraging. apidologie 34: 333-337.

27. van den Eijnde J., A. Ruijter \& J. van der Oteen. 1991. Method for rearing Bombus terrestris continuosly and the production of bumblebee colonies for pollination purposes. Act. Hort. 288: 154-158.

28. Velthuis H.W. \& A. van Doorn. 2006. A century of advances in bumblebee domestication and the economic and environmental aspects of its comercializartion for pollination. Apidologie, 37: 421-451.

29. Whittington R. \& M. Winston. 2004. Comparision and examination of Bombus occidentalis and Bombus impatiens (Hymenoptera: Apidae) in tomato greenhouses. J. Econ. Entomol. 97: 1384-1389.

30. Winter K., L. Adams, R. Thorp, D. Inouye, L. Day, J. Ascher \& S. Buchmann. 2006. Importation of Non-Native Bumble Bees into North America: Potential Consequences of Using Bombus terrestris and Other Non-Native Bumble Bees for Greenhouse Crop Pollination in Canada, Mexico, and the United States. North American Pollinator Protection Campaign (NAPPC). 1-33.

31. Zucchi, R. 1973. Aspectos bionômicos de Exomalopsis aureopilosa e Bombus atratus incluindo considerações sobre a evolução do comportamento social (Hymenoptera, Apoidea). Ph.D. Thesis, Faculdade de Filosofia, Ciências e Letras de Ribeirão Preto, São Paulo, Brasil.

25. Silva-Matos E. V. \& C.A. Garófalo. 1995. Observations on the development of queenless 